that meet this standard of blinding and placebo effects potentially affect the estimates. One example of a study where it was impossible to blind the participants is the Heating Housing and Health Study (HHHS). Here the intervention was the installation of a modern efficient heater in the participants' homes.

Methods Using data from the HHHS, we explored three different approaches to estimate the placebo effect: (1) Dummy outcome variables (where we examined variables similar to the real outcomes, on which the intervention is known to have no effect); (2) identifying variables that may indicate a high susceptibility to placebo effects; and (3) modelling the effects of measured intermediate variables (in the heating example the direct effect of the intervention should be associated with a rise in temperatures).

Results Combining these approaches in a Bayesian framework we calculated estimates of the placebo effect and intervention effects across a range of outcome measures in the HHHS. The changes in the estimates of these intervention effects showed which results were likely to be affected by placebo effects. These findings agree well with our expectations.

Conclusion With carefully chosen assumptions, it is possible to use data already collected and a Bayesian modelling approach to obtain informative estimates of likely placebo effects and hence provide better estimates of the true effects of an intervention in unblinded RCT's.

\section{P1-496 AN EXAMINATION OF THE PSYCHOMETRIC PROPERTIES OF THE GHO-28 SUBSCALES IN A BI-ETHNIC ANTENATAL SAMPLE IN THE UK: PRELIMINARY RESULTS FROM THE BORN IN BRADFORD COHORT}

doi:10.1136/jech.2011.142976g.84

\author{
${ }^{1} \mathrm{~S}$ Prady, ${ }^{* 2} \mathrm{~J}$ Miles, ${ }^{3} \mathrm{~L}$ Fairley, ${ }^{1} \mathrm{~K}$ Bloor, ${ }^{1} \mathrm{~S}$ Gilbody, ${ }^{1} \mathrm{~K}$ Kiernan, ${ }^{1} \mathrm{~K}$ Pickett, ${ }^{3} \mathrm{P}$ Raynor, \\ ${ }^{3} \mathrm{~J}$ Wright. ${ }^{1}$ University of York, York, UK; ${ }^{2}$ Unaffiliated, USA; ${ }^{3}$ Bradford Institute of \\ Health Research, Bradford, UK
}

Introduction Born in Bradford is a large multi-ethnic birth cohort drawn from the North of England. As background to comparing mental health across our sample we conducted a confirmatory factor analysis (CFA) and invariance testing for each of the four-factor seven-item subscales of the GHQ-28 for a bi-ethnic subpopulation.

Methods We examined data from White $(\mathrm{N}=2011)$ and Pakistaniorigin ( $N=1516)$ women who responded to the GHQ-28 in English. We fitted a single factor model to data from each subscale to the White subpopulation and tested for invariance against the Pakistani women's data. Where configural invariance was indicated for a proposed factor we followed a model generation strategy for each subpopulation and tested for invariance on this reduced item set.

Results In general, the models gave poor fit. Fit was acceptable when we reduced the number of items from seven to four for the Somatic, Anxiety/Insomnia and Severe Depression subscales. The unmodified Social Dysfunction subscale fitted both groups adequately. After correcting for differential item functioning (Item 4, Felt ill) we found Pakistani women were more likely to have worse scores on the reduced Somatic subscale $(d=0.10, p<0.001)$. They also had worse scores on the unmodified Social Dysfunction $(d=0.03, p<0.001)$ and reduced Severe Depression subscales $(d=0.14$ $p<0.001)$. Severe fit difficulties were observed for the Anxiety/ Insomnia subscale.

Conclusion The unmodified GHO -28 subscales did not fit a CFA model well and modifications were needed to ensure measurement equivalence. Pakistani women in this preliminary dataset fared significantly worse on all measureable subscales than White women.

\section{P1-497 SUPPORT AND ADMINISTRATION OF THE MULTI-SITE GLOBAL HEALTH INITIATIVE PROGRAM OF COMBATING NON-COMMUNICABLE CHRONIC CARDIOVASCULAR AND PULMONARY DISEASES IN DEVELOPING COUNTRIES}

doi:10.1136/jech.2011.142976g.85

${ }^{2} \mathrm{H} Z$ Oian, ${ }^{1} \mathrm{R}$ N N Dianis, ${ }^{1} \mathrm{~T}$ Wolbach, ${ }^{3} \mathrm{~J}$ Bolognese. ${ }^{1}$ Westat, Rockville, Maryland, USA; ${ }^{2}$ Vanderbilt University, Nashville, Tennessee, USA; ${ }^{3}$ Westat, Rockville, Maryland, USA

The National Heart, Lung and Blood Institute (NHLBI) teamed with United Health Group's Chronic Disease Initiative (CDI) to reduce the burden of noncommunicable cardiovascular and pulmonary diseases (CVPD) by building research and training capacities at 11 emerging Centers of Excellence (COEs) in Argentina, Bangladesh, China, Guatemala, India (Bangalore), India (New Delhi), Kenya, Peru, South Africa, Tunisia and the USA Mexico Border. To support diverse program needs and provide logistical expertise, Westat Company serves as the administrative coordinating center (ACC) for NHLBI's Global Health Initiative (GHI). Maintaining a global network poses challenges at the country and international levels, but is an opportunity for COEs to share their experiences and consider future collaborations to minimise redundancies in effort. The ACC manages these global discussions by identifying communication options suited to each country's technological capabilities. The ACC also organises semi-annual network meetings; reviews site establishment, protocol registration, human subjects protection (HSP) and good clinical practice (GCP) training documents; collects data to track program achievements; and maintains a web site to post documents, announcements and discussion forums. COEs are currently conducting research activities to classify, quantify, reduce and/or prevent risk factors associated with noncommunicable, chronic CVPD. While the research activities are designed to address local needs, the findings should also influence global health policy. We will present the experiences of providing administrative, operational, or technical assistance to the research and training activities of these COEs, and describe the high impact projects-in-progress at the COEs and the ACC.

\section{P1-498 MEASURES AND MODELS FOR CAUSAL INFERENCE IN CROSS-SECTIONAL STUDIES: ARGUMENTS FOR THE APPROPRIATENESS OF THE PREVALENCE OR AND RELATED LOGISTIC REGRESSION}

doi:10.1136/jech.2011.142976g.86

${ }^{1} \mathrm{M}$ Reichenheim, ${ }^{*} \mathrm{E}$ Coutinho. ${ }^{1}$ Department of Epidemiology, Institute of Social Medicine (IMS), Rio de Janeiro State University (UERJ), Rio de Janeiro, Brazil; ${ }^{2}$ Department of Epidemiology and Quantitative Methods, National School of Public Health Sergio Arouca (ENSP), Oswaldo Cruz Foundation (Fiocruz), Rio de Janeiro, Brazil

Introduction Several papers have discussed which effect measures are appropriate to capture the contrast between exposure groups in cross-sectional studies, and which related multivariate models are suitable. Although some have favoured the Prevalence Ratio over the Prevalence OR-thus suggesting the use of log-binomial or robust Poisson instead of the logistic regression models - this debate is still far from settled and requires close scrutiny.

Method In order to evaluate how accurately true causal parameters such as Incidence Density Ratio (IDR) or the Cumulative Incidence Ratio (CIR) are effectively estimated, we present a series of scenarios in which a researcher happens to find a preset ratio of prevalences (eg, 2.0) in a given cross-sectional study.

Results Provided essential and non-waivable structuring conditions for causal inference are all met, results show that the CIR is most often inestimable whether through the prevalence ratio or the 